\title{
Design and performance evaluation of narrowband rectangular optical filter based on stimulated Brillouin scattering in fiber
}

\author{
Invited Paper
}

\author{
Lilin $\mathrm{Yi}^{1}{ }^{1}$ - Wei Wei ${ }^{1} \cdot$ Mengyue Shi $^{1} \cdot$ Yves Jaouen $^{2} \cdot$ Weisheng Hu${ }^{1}$
}

Received: 5 April 2015 / Accepted: 11 June 2015 / Published online: 4 August 2015

(C) The Author(s) 2015. This article is published with open access at Springerlink.com

\begin{abstract}
We propose a rectangular optical filter based on stimulated Brillouin scattering (SBS) in fiber with tunable bandwidth from $50 \mathrm{MHz}$ to $4 \mathrm{GHz}$ at $15-\mathrm{MHz}$ tuning resolution. The steep-edged rectangular shape of the filter is precisely controlled utilizing digital feedback compensation of the multi-tone pump light. The passband ripple is $\sim 1 \mathrm{~dB}$ by nonlinearity management of the pump light and using the fiber with a single Brillouin peak. The filter selectivity is improved to more than $40 \mathrm{~dB}$ by using pump-splitting dualstage configuration. We analyze the noise performance of the proposed SBS filter and demonstrate a sub-band extraction of a multi-band orthogonal frequency division multiplexing (OFDM) signal. Furthermore, we validate the amplification performance with different gains for OFDM signal, which shows the potential capability of the filter in the fields of optical signal processing.
\end{abstract}

Keywords Rectangular optical filter · Stimulated Brillouin scattering $\cdot$ Optical signal processing

\section{Introduction}

A tunable rectangular filter with an ultra-flat passband and steep edges brings about many benefits. The flat passband will not distort the signal thus keeping high signal fidelity. The steep edges can suppress interference from adjacent

Lilin Yi

lilinyi@sjtu.edu.cn

1 State Key Lab of Advanced Optical Communication Systems and Networks, Shanghai Jiao Tong University, Shanghai 200240, China

2 Institut Télécom/Télécom ParisTech, CNRS UMR 5141, 46 Rue Barrault, 75634 Paris, France bands at the extreme. And the tunability makes it more flexible to meet different requirements. Several methods have been proposed to implement such filters, including specially designed fiber Bragg gratings (FBG) [1], Fabry-Perot etalons [2] and stimulated Brillouin scattering (SBS) [3-6]. Among all the above methods, SBS is considered as one of the most promising techniques. The wavelength of the SBS-based filter can be tuned easily by changing the wavelength of the pump. The filter bandwidth and the shape can be also flexibly changed by controlling the pump spectrum using external phase modulation [3], direct current modulation [4] and cascaded phase and intensity modulation [5]. However, it is very difficult to control the pump spectrum precisely in the previous works; therefore, the exact flat top and steep edges as the properties of ideal rectangular filter are hardly achieved.

In this paper, we present a narrowband rectangular SBS filter with tunable bandwidth from $50 \mathrm{MHz}$ to $4 \mathrm{GHz}$ at 15 $\mathrm{MHz}$ tuning resolution. We modulate the pump using external intensity modulation by an electrical frequency comb whose amplitude and initial phase can be digitally controlled accurately. A feedback compensation process is proposed to revise the pump shape precisely. Some preliminary results have been presented in a previous paper [7] which shows the validity of the feedback method. On the foundation of the feedback process, we further propose the nonlinearity management to mitigate the four-wave mixing (FWM) effect and utilize a more suitable fiber with a single Brillouin gain peak to increase the filter flatness [8]. As a result, the inband ripple is decreased to $\sim 1 \mathrm{~dB}$ for all bandwidth cases, while the unwanted out-of-band gain is further suppressed. The out-of-band SBS gain originated from the FWM effect of the multiple pump lines will degrade the filter selectivity. We propose a pump-splitting dual-stage confirmation to improve the filter selectivity to more than $40 \mathrm{~dB}$. Based on this filter, we analyze the noise performance and demonstrate 
a sub-band extraction of a multi-band orthogonal frequency division multiplexing (OFDM) signal; furthermore, we validate the SBS amplification with different gains for OFDM signal and prove the flexibility and validity of the filter.

\section{Rectangular filter}

The natural SBS gain is Lorentzian shape with bandwidth ranging from 10 to $50 \mathrm{MHz}$ depending on different types of fibers [9]. Theoretically, in order to obtain the ideal rectangular SBS gain spectrum, a pump consisting of equalamplitude spectral lines with interval equaling the natural SBS gain bandwidth is required. Instead of controlling the pump spectrum in the time domain, we firstly generate electrical frequency combs with equal amplitude at each spectral line using an arbitrary waveform generator (AWG). Then, we modulate them with optical carrier-suppressed single-sideband (OCS-SSB) modulation to generate optical frequency combs as the Brillouin pump. The SBS filter bandwidth adjustment is achieved simply by controlling the number of electrical spectral lines so as to the pump spectral lines. In addition, we can control the amplitude, the initial phase of each electrical spectral line and the frequency spacing of spectral lines digitally and precisely. It guarantees that there are no spectral components out of the comb region at all, resulting in a steep-edged pump light; thus, the SBS gain spectrum obtained has very steep edges as well, which is unachievable in the previously reported schemes.

Since the SBS gain increases with the pump power exponentially, even little pump power drifting results in great gain difference. However, on account of the nonlinear responses of electrical and optical components such as the optical modulator and its driver, the flat electrical spectral lines lead to uneven SBS gain inevitably as sketched in Fig. 1. Thus, we propose an effective feedback compensation scheme as follows: firstly generate the original pump lines by OCS-SSB modulation using flat electrical spectral lines. Then measure the SBS gain spectrum by an electrical vector network analyzer (EVNA) and calculate the new amplitude of each electrical spectral line. Taking the linear approximation error and the non-ideal responses of electrical and optical components into account, a rectangular gain spectrum can only be obtained by repeating the processes of generation, measurement and feedback process successively for several times.
Once the feedback compensation is completed, the electrical spectral lines can be recorded for rectangular SBS filter generation.

In the feedback process, it is assumed that the SBS gain at a certain frequency is only related to the corresponding electrical spectral line. It works well when the filter bandwidth is narrow. However, with the increasing of the filter bandwidth, the increasing pump power and number of spectral lines cause remarkable FWM in the optical fiber which redistributes the pump power and generates new spectral lines at the stop band. Another factor not taking into account is the nonlinearity of the modulation such as third-order intermodulation that also introduces new spectral lines and cannot be neglected when the pump power is high. The nonlinearityinduced spectral components are sketched in Fig. 2a, b in the color of yellow. It results in a partial failure of the feedback process, leading to larger passband ripple and out-of-band gain. Therefore, an effective nonlinearity management is required.

We propose a nonlinearity management method as shown in Fig. 2c. We set frequency interval of the electrical spectral lines randomly around the natural SBS gain bandwidth instead of the equal interval, i.e., 14, 15 and $16 \mathrm{MHz}$. In this case, the new spectral lines generated by the FWM and the nonlinear modulation are no longer superposing on the original lines. As a result, the power of these new spectral lines is very small compared with the original lines and can just induce tiny gain or even under the threshold of SBS effect.

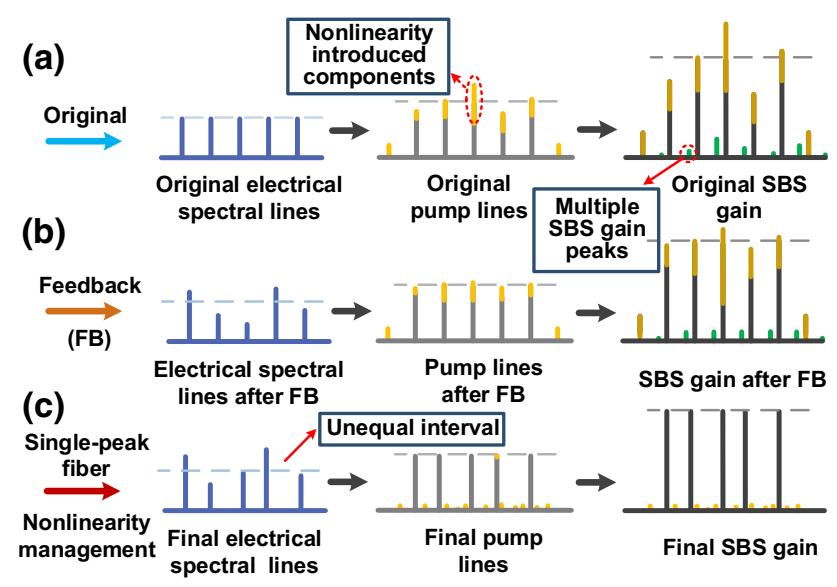

Fig. 2 The relationship between electrical spectral lines, pump lines and the SBS gains in different cases
Fig. 1 Feedback compensation scheme

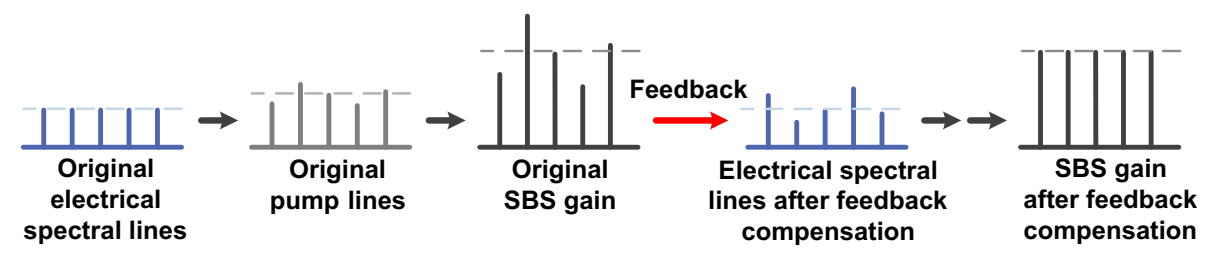


Fig. 3 The natural SBS gain in a G-652 fiber, b fiber with a single Brillouin peak

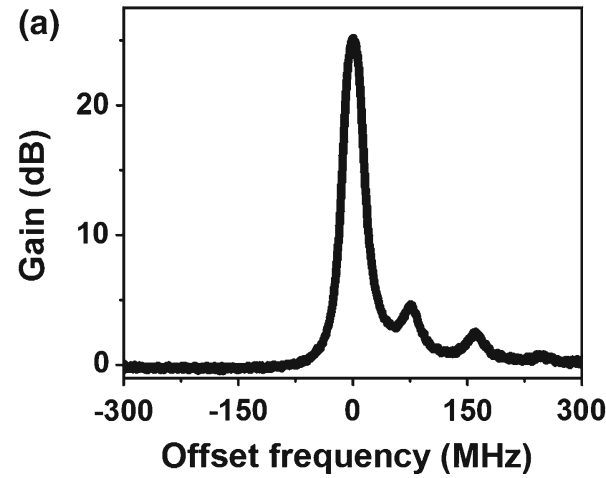

Therefore, the flatness of the passband gain can be greatly improved, and the unwanted out-of-band gain can be partly suppressed as well.

Moreover, the natural SBS gain spectrum is related to the material doping profile [10] and the structure of the fiber [11]. In the Germanium-doped G-652 fiber that we used in the previous work [7], there are 4 SBS peaks (i.e., four first Lom acoustic modes) as sketched in Fig. 2a, b in the color of green, while the measured result is shown in Fig. 3a. The multiple minor SBS gain peaks superpose with other main SBS gain peaks generated by different pump lines and will affect the feedback process. It contributes to the un-flat filter passband and induces out-of-band gain as well. Therefore, we employ a fiber with a single Brillouin peak (a standard single-mode fiber in our lab) as shown in Fig. 3b which is highly desired in our experiment. By employing both the nonlinear management and the new fiber with single Brillouin peak, the final SBS gain will be ultra-flat as sketched in Fig. 2c.

The experimental setup is shown in Fig. 4. An external cavity laser (ECL) operating at $1550 \mathrm{~nm}$ is split into two branches. In the upper branch, an AWG is used to generate the electrical spectral lines with random frequency intervals within $\pm 1-\mathrm{MHz}$ deviation from the natural SBS bandwidth of $15 \mathrm{MHz}$, i.e., 14,15 and $16 \mathrm{MHz}$. Then it is modulated on the light to generate the SBS pump lines utilizing a dual-parallel Mach-Zehnder modulator (DPMZM). With proper phase control of the driving signals and bias con- trol of the DPMZM, the OCS-SSB modulation is achieved. The OCS-SSB signal is then amplified by an erbium-doped fiber amplifier (EDFA) and launched into the $12.5 \mathrm{~km}$ singlepeak fiber through an optical circulator. In the lower branch, a sweeping signal covering the whole SBS gain region from an EVNA is modulated on the light to generate the probe signal. An optical bandpass filter (BPF) removes the left sideband of the probe signal for stable SBS gain measurement. Then the probe light goes through the single-peak fiber and is amplified once it sweeps within the SBS gain region. A polarization controller (PC3) is used to achieve the maximum SBS gain. After the SBS process, the probe signal is detected by a photodiode (PD) and then sent into the EVNA.

The measured SBS spectra with 3-GHz bandwidth in different cases are shown in Fig. 5. The original SBS gain generated by the electrical spectral lines with equal amplitude and equal frequency interval is seriously affected by the FWM and nonlinear response of the electrical and optical components. As shown in Fig. 5a, the SBS gain is far from the rectangular shape. When implementing the feedback compensation as shown in Fig. 5b, the ripple is still as high as $3.70 \mathrm{~dB}$ with large unwanted gain out of the passband. After utilizing the unequal interval spectral lines, the passband ripple in Fig. $5 \mathrm{c}$ is significantly reduced to $1.56 \mathrm{~dB}$. By using the single-peak fiber, the ripple is further decreased to $1.00 \mathrm{~dB}$ as shown in Fig. $5 \mathrm{~d}$, and the gain at stop band is smaller than the previous cases.
Fig. 4 Experimental setup. Inset $(i)$ single-sideband pump $f_{\mathrm{p}}$, SBS gain around $f_{\mathrm{g}}$, and ECL laser frequency $f_{\mathrm{c}}$, (ii) sweeping probe signal, (iii) sweeping probe signal amplified by the SBS gain

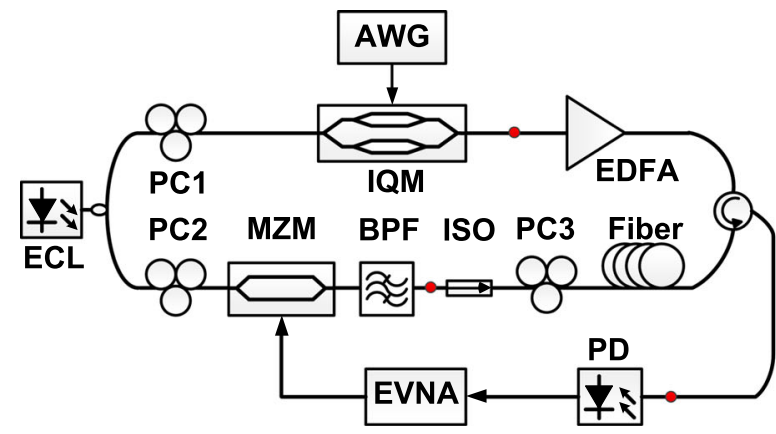

(i)

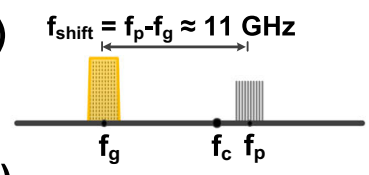

(ii)

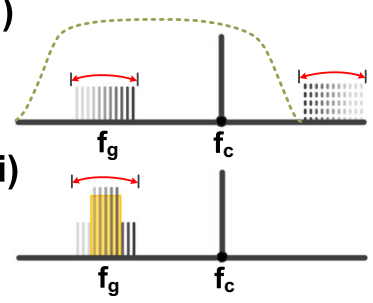


Fig. 5 The measured SBS spectra at different cases
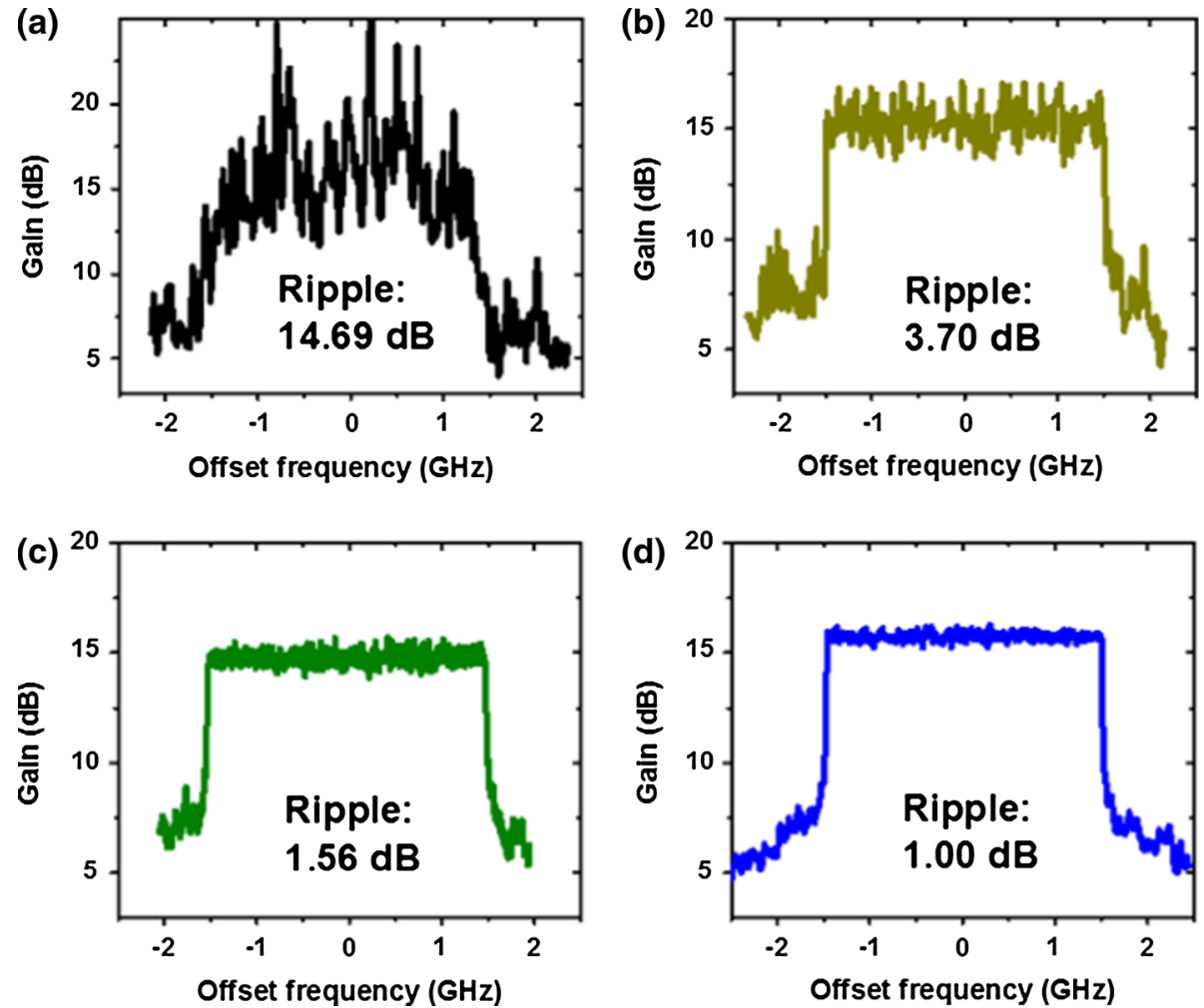

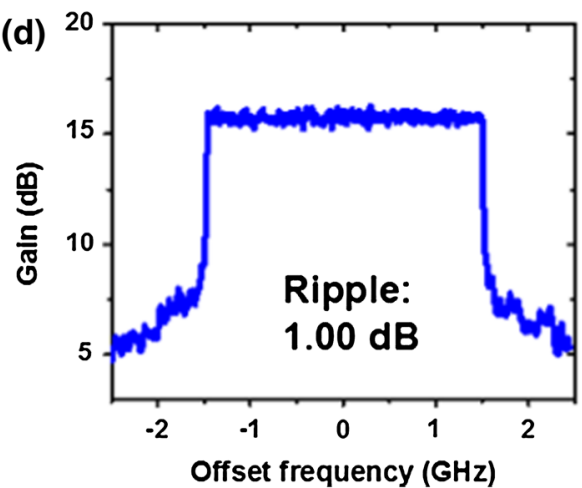

The bandwidth of the proposed filter can be changed flexibly with a resolution of $15 \mathrm{MHz}$ by changing the number of the electrical spectral lines generated by the AWG. The maximal rectangular SBS filter bandwidth is $4 \mathrm{GHz}$ with $12-\mathrm{dB}$ gain limited by the pump power.

\section{Filter selectivity improvement}

The filter selectivity cannot be increased by simply increasing pump power due to two reasons. The first reason is the competition between SBS and SRS effect. When the Brillouin pump power is higher than several hundreds of $\mathrm{mW}$, it starts acting as Raman pump at the same time which results in SBS pump power consumption. The second reason for filter selectivity limitation is FWM effect induced by multi-frequency comb. It generates new components out of the filter passband and cannot be completely mitigated by the nonlinearity management method. When the pump power increases, FWM effect is getting much more severe, especially in broad bandwidth cases. The out-of-band gain will consume considerable amount of pump power and disrupt the rectangular shape generation. Thus when the pump power continues to increase, most of the power is wasteful and brings about hardly no benefit.

In order to increase the filter selectivity, we propose a pump-splitting dual-stage scheme. Instead of using a single pump with high power, we split it into two stages and amplify the signal twice successively. Note that the filter selectivity is only related to the on-off gain and has nothing to do with the transmission loss; extra attenuation can be induced between the two stages to prevent the signal saturation in the second stage. In this case, the pump power of each stage is not too high to stimulate high Raman gain nor to induce too much out-of-band FWM components. Moreover, the decrease in pump power for each stage will decrease the induced noise from the spontaneous Brillouin emission [12]. Thus the filter selectivity can be increased dramatically, and better noise performance can be achieved at the same time.

The experimental setup is shown in Fig. 6. The process of generating SBS pump lines, sweeping signal, feedback compensation, as well as nonlinearity management is described as above. The difference in the OCS-SSB signal of the upper branch is then split into two parts equally and send into two identical 25-km-long SMFs, which are under the same strain and temperature conditions to ensure the same Brillouin characteristics. At each stage, a polarization controller (PC) is used to maintain the SBS gain at the maximum value. An optical attenuator (ATT) between the two stages can prevent signal saturation in the second stage therefore effectively increase the filter selectivity. The amplitude response sketched in Fig. 6(iv) is measured by the EVNA, and the SBS gain spectrum can be obtained by comparing the results between the SBS pump active or not.

The amplitude and phase responses of both single- and dual-stage filters are shown in Fig. 7. The pump power is set 


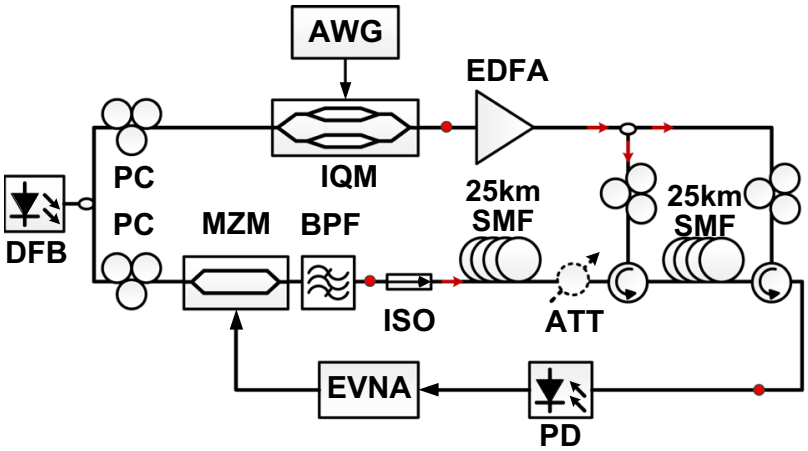

(i)

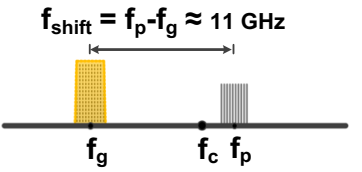

(iii)

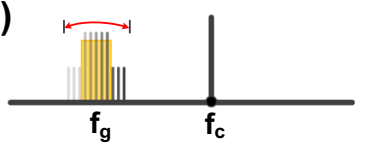

(ii)

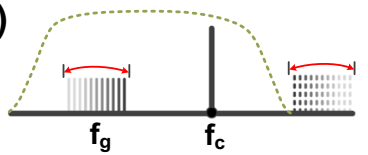

(iv)

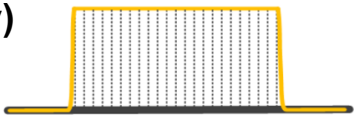

Fig. 6 Experimental setup. Inset (i) single-sideband pump $f_{\mathrm{p}}$, SBS gain around $f_{\mathrm{g}}$, and ECL laser frequency $f_{\mathrm{c}}$, (ii) sweeping probe signal, (iii) sweeping probe signal amplified by the SBS gain, (iv) measured gain spectrum

to 21,24 and $27 \mathrm{dBm}$ for filter bandwidth of 1,2 and $3 \mathrm{GHz}$, respectively. As shown in Fig. 7a, for all bandwidth cases, the filter gain has been improved obviously with the same total pump power, while the passband flatness remains at the same level. For the 3-GHz filter, the maximum gain of single-stage filter is limited to $\sim 18.5 \mathrm{~dB}$ due to SRS and the severe outof-band FWM components. By using the dual-stage scheme, the gain has been increased to $\sim 32 \mathrm{~dB}$. The high selectivity makes the filter shape more like rectangular than flat-top trapezoid. For the 1-GHz case, by using dual-stage configuration, the gain is increased while the out-of-band FWM effect is slightly mitigated. As to the phase response shown in Fig. 7b, all the curves are very smooth due to the small passband ripple, and higher gain of the dual-stage filter leads to larger phase variation compared with the single-stage case. The results have clearly proved the validity of the dual-stage scheme to increase the filter selectivity.

\section{System performance evaluation}

It is well-known that the Brillouin amplification is accompanied with strong noise [13]. We analyze the noise performance of the proposed SBS filter with the experimental setup shown in Fig. 8a. A 300-MHz single-frequency signal modulates the CW light from an external cavity laser (ECL). After passing through the active SBS filter and an optical attenu-
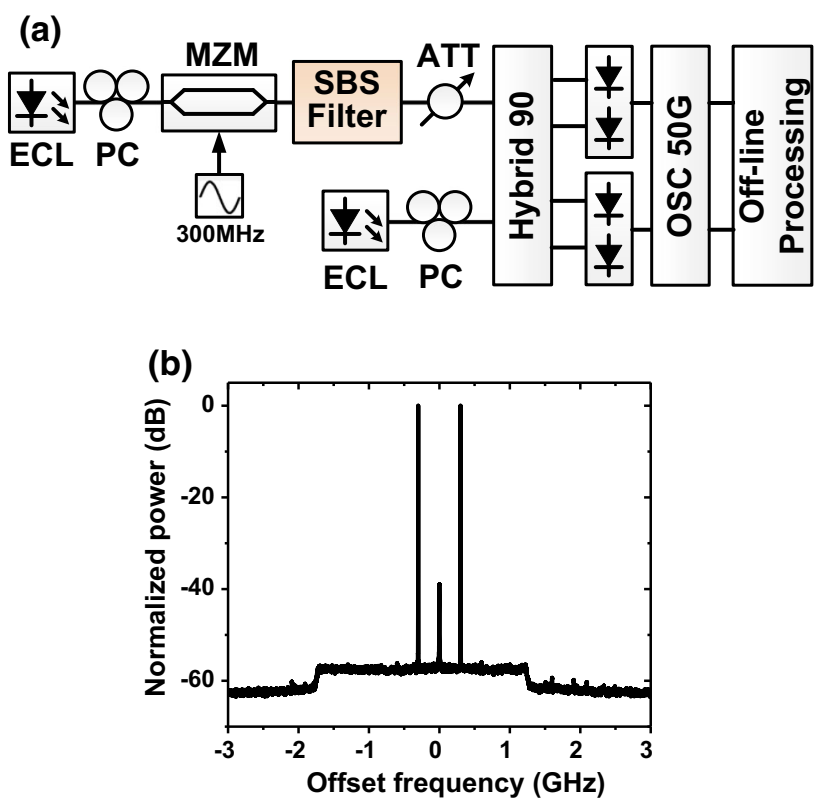

Fig. 8 a Noise measurement setup and $\mathbf{b}$ the electrical spectrum of the amplified signal by a $3-\mathrm{GHz}$ SBS filter

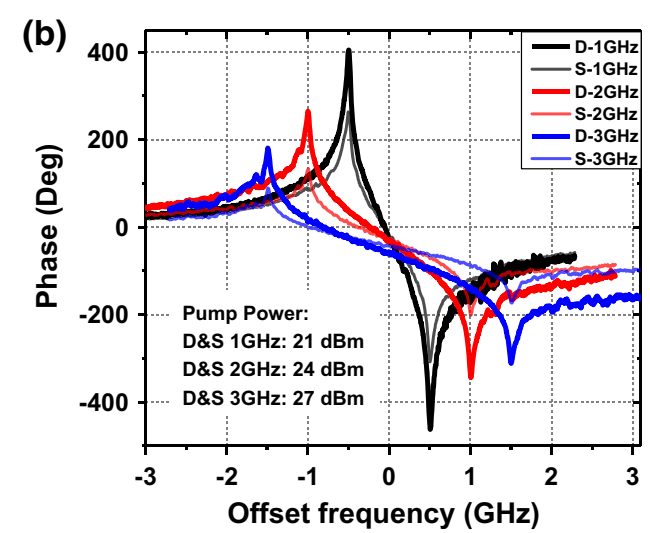

Fig. 7 Filter comparison between single- and dual-stage approaches. a The amplitude and $\mathbf{b}$ phase response of the two types of filter. $D$ dual stage, $S$ single stage 
ator to maintain the same power level, the signal is detected by a coherent receiver. Figure $8 \mathrm{~b}$ shows the spectrum of the SBS amplified signal which is calculated from the waveform obtained by a real-time oscilloscope from the receiver since we do not have an ultra-high-resolution optical spectrum analyzer for direct observation. The symmetric frequency line is due to the carrier-suppressed double-sideband modulation by the Mach-Zehnder modulator. We do not use the $\mathrm{CW}$ light as the signal since the DC noise from the oscilloscope itself is significant. The noise pedestal is linked to SBS gain which is shifted off-center due to slight frequency detuning between pump and signal waves.

Then the signal to noise ratio (SNR) is carefully measured. We use the amplified signal peak power and the average noise power in the SBS gain region to calculate the SNR. We define the SNR penalty at $1-\mathrm{GHz}$ bandwidth with 19 $\mathrm{dBm}$ pump power as reference since the SNR penalty under this condition is not obvious compared with the back-toback case. The relation between the pump power and the SNR is shown in Fig. 9a. We can conclude that the general trend of SNR penalty increases with the increase of the pump power, and the pump power level is the main factor to induce noise. For the single-stage filter, the turning point is around $20 \mathrm{dBm}$, and the three curves show strong consistency. As to the dual-stage filter, the turning points generally shift $2-3 \mathrm{~dB}$ higher in both the $2-\mathrm{GHz}$ and $3-\mathrm{GHz}$ cases, while in $1-\mathrm{GHz}$ case, the turning point remains the same. So if we can improve the pump efficiency to reduce the required pump power for the same gain value, the SNR performance can be improved. From this regard, the pump-splitting dualstage configuration is preferred. Figure $9 \mathrm{~b}$ demonstrates the SNR variation with different SBS gains. We can observe steep rise in SNR penalty with the increase in SBS gain after a certain turning point for both single- and dual-stage cases. For the small gain cases, the dual-stage-induced SNR penalty is only $1-2 \mathrm{~dB}$. Once the gain reaches 32,19 , and
$13 \mathrm{~dB}$ for the bandwidth of 1,2 , and $3 \mathrm{GHz}$, respectively, the noise performance of the dual-stage scheme is always better than the single-stage method. If $10-\mathrm{dB}$ attenuation between the two stages is used, the SNR will degrade when the gain is small, but when the gain reaches $34.5 \mathrm{~dB}$, it induces smallest penalty and becomes the best choice. Note that for a SBS-based microwave photonic filter, high selectivity with low noise is preferred; thus, the pump-splitting dualstage approach is a simple but effective method to reach this goal.

After evaluating the noise performance of the SBS-based filter, we demonstrate a sub-band extraction of a multi-band orthogonal frequency division multiplexing (OFDM) signal using the pump-splitting dual-stage SBS filter. The experimental setup consists of three parts: the transmitter, the SBS-based filter and the coherent receiver as shown in Fig. 10. In the transmitter part, the light from an external cavity laser (ECL) and two distributed feedback lasers (DFB) operating at $\sim 1543 \mathrm{~nm}$ are modulated by the electrical OFDM signal. As only a single output of the Tektronix7221B AWG is available for OFDM signal generation, an OFDM signal satisfying the Hermitian symmetry is generated. This constraint does not affect our analysis as all sub-carriers are treated independently at the receiver side. Given the instability of the three lasers, the minimum band gap of the three sub-bands is set to $300 \mathrm{MHz}$. For each sub-band, 128 sub-carriers are used in order to mitigate the effect of the ECL phase noise of $\sim 100 \mathrm{KHz}$. Both quadrature-phase-shiftkeying (QPSK) and 16-quadrature-amplitude-modulation (16-QAM) formats are employed for each sub-carrier at the sampling rate of $2.5 \mathrm{GSamples} / \mathrm{s}$. The bandwidth is set to $2 \mathrm{GHz}$ by adjusting the number of empty sub-carriers. After passing through an isolator (ISO) to block inverse pump light, the three OFDM sub-bands are launched into the pumpsplitting dual-stage SBS filter with bandwidth of $2.2 \mathrm{GHz}$, where the extra $200 \mathrm{MHz}$ bandwidth is dedicated to laser
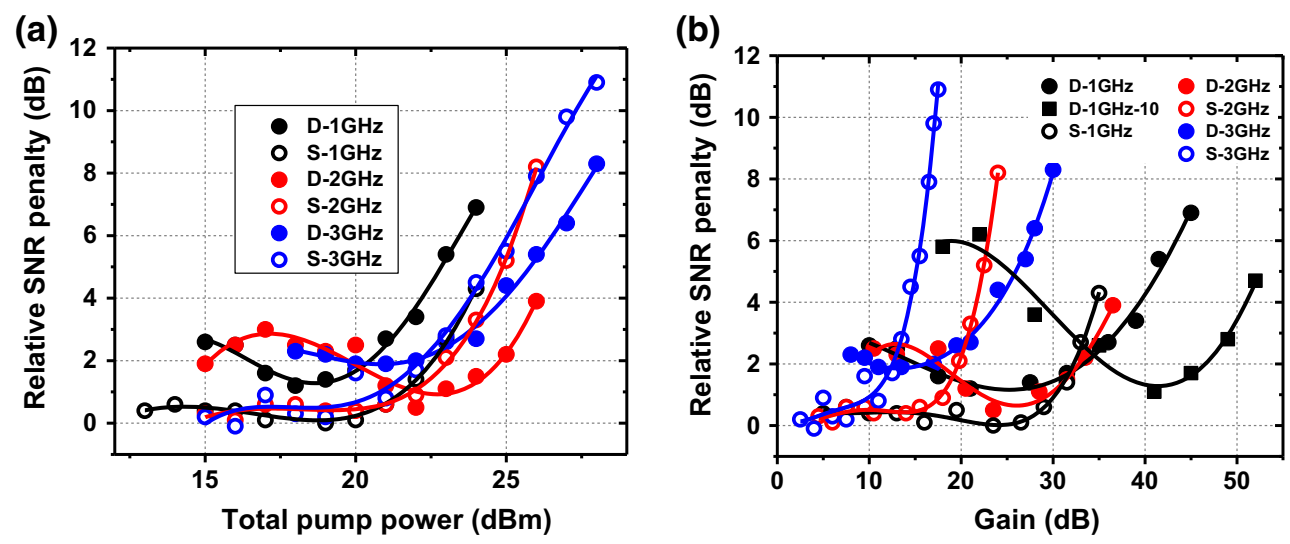

Fig. 9 Noise comparison between the single and cascade filters, a the relation between SNR and pump power, $\mathbf{b}$ the relation between SNR and SBS gain. $D$ dual stage, $S$ single stage. “- $10 "$ means $10 \mathrm{~dB}$ optical attenuation between two stages 


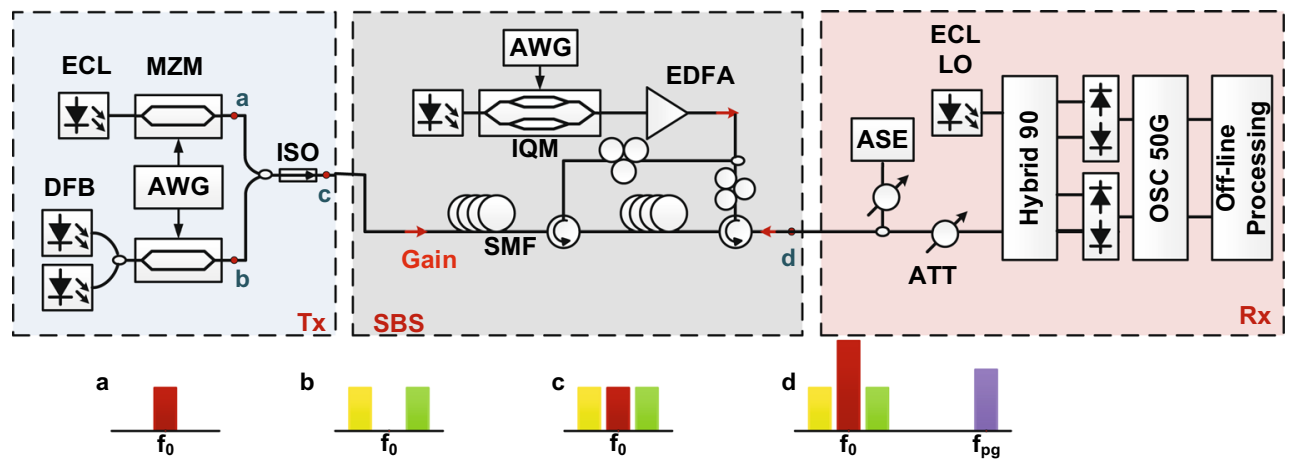

Fig. 10 Experimental setup and the optical spectrum schemes at different points
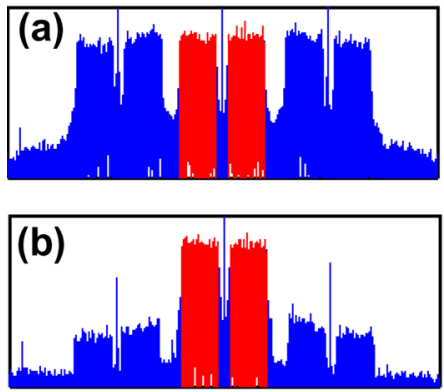
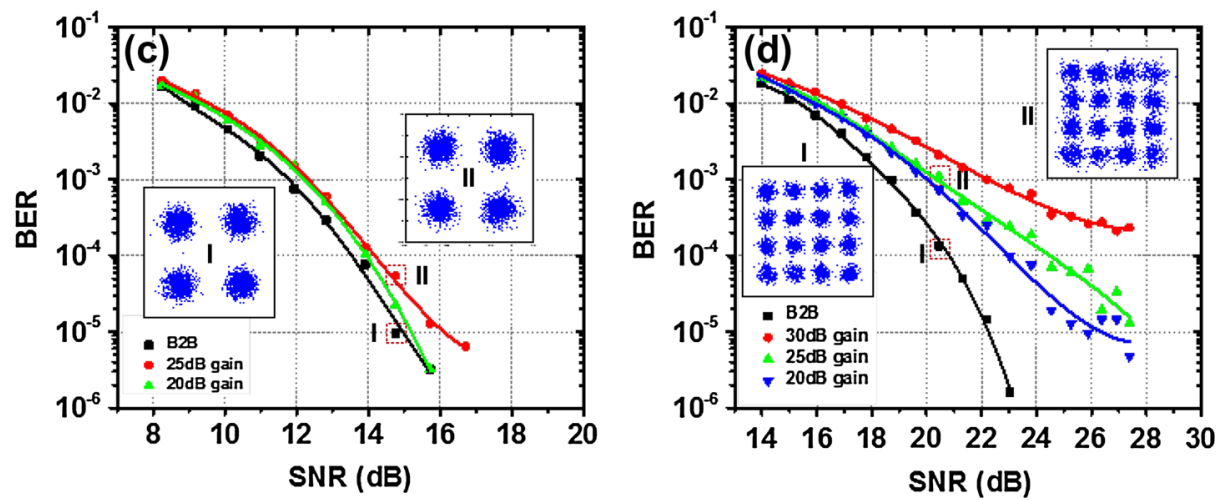

Fig. 11 Amplified OFDM signals: a original 3 sub-bands OFDM signal, b amplified central band by using the SBS gain filter, c BER-SNR performance for QPSK, d BER-SNR performance for 16-QAM

wavelength drift mitigation. The amplified central sub-band from the SBS filter is adjusted to the optimal power of the receiver, and a broadband ASE noise source is added for the SNR-BER measurement. Finally the OFDM signal is detected by an intradyne coherent receiver followed by a high-speed real-time oscilloscope. QPSK and 16-QAM constellations are then obtained by off-line processing. A more precise description of OFDM signal generation and detection is in $[14,15]$.

The validity of the SBS amplification with different gains for OFDM signal has been assessed. Figure 11a shows the electrical spectrum of the received original 3-band OFDM signal, and Fig. 11b illustrates the spectrum when the central band is amplified by the SBS gain filter. The SNR-BER curves for the QPSK format are presented in Fig. 11c while the 16-QAM case in Fig. 3d. Different constellation diagrams are also given in the insets. After being amplified by the SBS gain filter with $25-\mathrm{dB}$ gain, the SNR penalties are only $\sim 0.5$ and $\sim 2 \mathrm{~dB}$ at a BER of $10^{-3}$ for QPSK and 16QAM, respectively. For 16-QAM, larger penalty has been observed when the SNR is high in large gain cases because of the large SBS-ASE noise. The results have proved that the SBS gain induced penalty is not significant, especially when the signal format is QPSK. It also validates the feasibility of the proposed rectangular SBS gain filter in the OFDM system.

\section{Conclusion}

We have presented an ultra-flat rectangular optical filter based on stimulated Brillouin scattering and demonstrated a typical filter application of OFDM sub-band extraction and amplification. With flexible bandwidth from $50 \mathrm{MHz}$ to $4 \mathrm{GHz}$ and high tuning resolution of $15 \mathrm{MHz}$, only small penalties are induced by the SBS-ASE noise which prove the feasibility of the SBS amplification in OFDM systems. The proposed steep-edged rectangular filter can find versatile applications in optical and microwave signal processing.

Acknowledgments This work is supported by 973 Program (2012CB315602), National Nature Science Foundation of China (61322507 and 61132004) and Program of Excellent Ph.D. in China (201155).

Open Access This article is distributed under the terms of the Creative Commons Attribution 4.0 International License (http://creativecomm ons.org/licenses/by/4.0/), which permits unrestricted use, distribution, and reproduction in any medium, provided you give appropriate credit to the original author(s) and the source, provide a link to the Creative Commons license, and indicate if changes were made. 


\section{References}

[1] Zou, X.H., Li, M., Pan, W., Yan, L.S., Azana, J., Yao, J.P.: Allfiber optical filter with an ultranarrow and rectangular spectral response. Opt. Lett. 38, 3096-3098 (2013)

[2] Pruessner, M.W., Stievater, T.H., Goetz, P.G., Rabinovich, W.S., Urick, V.J.: Cascaded integrated waveguide linear microcavity filters. Appl. Phys. Lett. 103, 11105 (2013)

[3] Tanemura, T., Takushima, Y., Kikuchi, K.: Narrowband optical filter, with a variable transmission spectrum, using stimulated Brillouin scattering in optical fiber. Opt. Lett. 27, 1552-1554 (2002)

[4] Zadok, A., Eyal, A., Tur, M.: Gigahertz-wide optically reconfigurable filters using stimulated Brillouin scattering. J. Lightwave Technol. 25, 2168-2174 (2007)

[5] Sakamoto, T., Yamamoto, T., Shiraki, K., Kurashima, T.: Low distortion slow light in flat Brillouin gain spectrum by using optical frequency comb. Opt. Express 16, 8026-8032 (2008)

[6] Wise, A., Tur, M., Zadok, A.: Sharp tunable optical filters based on the polarization attributes of stimulated Brillouin scattering. Opt. Express 19, 21945-21955 (2011)

[7] Wei, W., Yi, L., Zhang, Y., Jaouen, Y., Song, Y., Dong, Y., Hu, W.: A bandwidth-tunable narrowband rectangular optical filter based on stimulated Brillouin scattering. In: Optical Fiber Communication Conference, OSA Technical Digest (online) (Optical Society of America, 2014), paper W4F.5

[8] Wei, W., Yi, L., Jaouën, Yves: et al.: An Ultra-Flat Rectangular Optical Filter Based on Stimulated Brillouin Scattering in Fiber, ECOC'2014, paper P.1.7

[9] Mamdem, Y., Burov, E., de Montmorillon, L., Jaouën, Y., Moreau, G., Gabet, R., Taillade, F.: Importance of residual stresses in the Brillouin gain spectrum of single mode optical fibers. Opt. Express 20, 1790-1797 (2012)

[10] Jaouën, Y., Canat, G., Sikali-Mamdem, Y., Gabet, R., Lombard, L., Burov, E.: Stimulated Brillouin scattering in specialty optical fibers: importance of material, structure and manufacturing parameters. In: Conference on Lasers and Electro-Optics 2012, OSA Technical Digest (online) (Optical Society of America, 2012), paper CF3N.1

[11] Yeniay, A., Delavaux, J.M., Toulouse, J.: Spontaneous and stimulated Brillouin scattering gain spectra in optical fibers. J. Lightwave Technol. 20, 1425-1432 (2002)

[12] Olsson, N., Van Der Ziel, J.: Characteristics of a semiconductor laser pumped Brillouin amplifier with electronically controlled bandwidth. J. Lightwave Technol. 5, 147-153 (1987)

[13] Tkach, R.W., Chraplyvy, A.R., Derosier, R.M.: Performance of a WDM network based on stimulated Brillouin scattering. IEEE Photonics Technol. Lett. 1, 111-113 (1989)

[14] Pincemin, E., Song, M., Karaki, J., Zia-Chahabi, O., Guillossou, T., Grot, D., Thouenon, G., Betoule, C., Clavier, R., Poudoulec, A., Van der Keur, M., Jaouën, Y., Le Bidan, R., Le Gall, T., Gravey, P., Morvan, M., Dumas-Feris, B., Moulinard, M., Froc, G.: Multi-band OFDM transmission at 100 Gbps with sub-band optical switching. J. Lightwave Technol. 32, 2202-2219 (2014)

[15] Awwad, E., Jaouën, Y., Othman, G.R.-B., Pincemin, E.: Polarization-time coding for PDL mitigation long-haul PolMux OFDM systems. Opt. Express 21, 22773-22790 (2013)

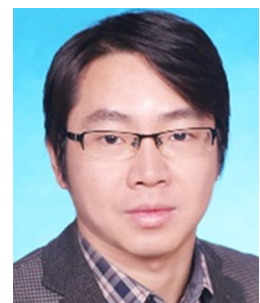

Lilin Yi has received the B.S. (2002) and M.S. (2005) from Shanghai Jiao Tong University (SJTU), China. He achieved the Ph.D. degree from Ecole Nationale Supérieure des Télécommunications (ENST, currently named as Telecom ParisTech), France and SJTU, China on March and June 2008, respectively, as a joint-educated $\mathrm{PhD}$ student. After graduation, he worked at Oclaro R\&D center as a product development manager and presided projects of Alcatel-Lucent 100G novel optical amplifier and Avanex next generation optical amplification platform. Since 2010, he joined the State Key Laboratory of Advanced Optical Communication Systems and Network, SJTU. Currently he is working as an associate professor. His main research topics include novel optical access networks, optical signal processing, graphene photonics and secure optical communications. Dr. Lilin Yi is the author or coauthor of more than 100 papers in peer-reviewed journals and conferences, including more than 10 invited papers, which have been cited more than 800 times (Google Scholar). Dr. Yi has achieved the awards of "National excellent $\mathrm{PhD}$ thesis in China" and "National Science Fund for Excellent Young Scholars of China."

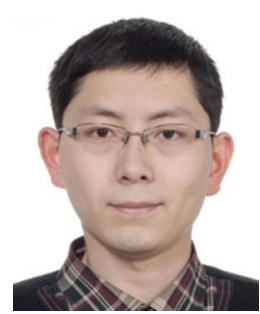

Wei Wei is a Ph.D. candidate at Shanghai Jiao Tong University (SJTU). He received his B.S. (2012) and M.S. (2015) from SJTU and continues his study for a Ph.D. degree in the State Key Laboratory of Advanced Optical Communication Systems and Networks. His research interest is optical signal processing based on stimulated Brillouin scattering.

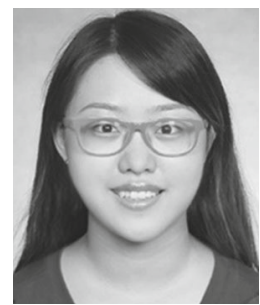

Mengyue Shi received her bachelor degree in electronic science and technology from Department of Optoelectronic Information in the University of Electronic Science and Technology of China (UESTC) in 2014. She is now pursuing her Ph.D. degree in information and communication engineering in the State Key Lab of Advanced optical communication systems and networks. Her main research field is optical signal processing based on stimulated Brillouin scattering. 


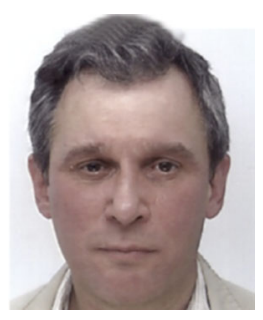

Yves Jaouen received his Ph.D. degree in Physics from Ecole Nationale Supérieure des Télécommunications (ENST), Paris, France in 1993 and then his HDR (Research management certificate) in 2003. He had joined ENST (now called TELECOM ParisTech) in the Communications and Electronic Department in 1982 where he is currently professor. He is lecturing in the domain of electromagnetic fields, optics and optical communications systems. His present researches include high bit rate coherent optical communication systems including digital signal processing aspects, new characterization techniques for advanced photonic devices, high power fiber lasers, fiber optics and remote sensing. He is author or co-author of more than 180 papers in journals and communications.

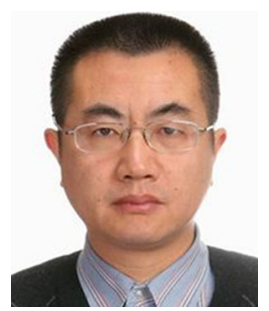

Weisheng Hu received B.S. (86), M.S. (89), and Ph.D. (94) from Tsinghua, Beijing University of Science and Technology (BUST), and Nanjing University. He joined Wuhan University of Science and Technology as assistant professor in 1989-1994, SJTU as postdoctorate fellow in 1997-1999 and as professor in 1999. He was director of the State Key Lab of Advanced Optical Communication Systems and Networks (20032007), member of coordinate task force of CAINONet and 3Tnet (1999-2006) and technology forecast of Shanghai (2004-). He serves TPC for OFC, APOC, Optics East, LEOS/PS, CLEO/PS, ICICS, and editorial board for JLT, COL and FOC. He led and participated 32 grants supported by NSFC, 863, MOE and Shanghai. He received one National Award and three Provincial/Ministry Awards for Science and Technology Progress. 